\title{
LncRNA GAS5 suppresses ER stress-induced apoptosis and inflammation by regulating SERCA2b in HG-treated retinal epithelial cell
}

\author{
LEI JIANG, CUN WANG and XINYUE SHEN \\ Department of Ophthalmology, The Third People's Hospital of Wuxi, Wuxi, Jiangsu 214000, P.R. China
}

Received October 31, 2019; Accepted April 15, 2020

DOI: $10.3892 / \mathrm{mmr} .2020 .11163$

\begin{abstract}
Hyperglycemia impairs the retinal functions in patients with diabetic retinopathy (DR). Downregulation of long non-coding RNA growth arrest-specific transcript 5 (lncRNA GAS5) expression in diabetes affects glucose intake and insulin signaling. Sarcoplasmic/endoplasmic reticulum $\mathrm{Ca}^{2+}$ ATPase 2 (SERCA2) mediates the regulation of endoplasmic reticulum (ER) stress and apoptosis in high glucose (HG)-treated podocytes. Therefore, the present study aimed to investigate the roles of IncRNA GAS5 and SERCA2 in retinal pigment epithelium cells exposed to HG. GAS5 expression levels were detected using reverse transcription-quantitative PCR. In addition, the expression levels of SERCA2b, ER stress-related proteins, pro-inflammatory factors and apoptotic proteins were determined by western blot analysis, ELISA or flow cytometry. The results showed that HG treatment induced ER stress in ARPE-19 human adult retinal pigment epithelial cells by upregulating the expression levels of phosphorylated (p)-protein kinase R-like ER kinase, p-eukaryotic initiation factor $2 \alpha$, activating transcription factor 4 and CCAAT/enhancer-binding protein homologous protein. In addition, HG treatment induced apoptosis by increasing Bax, Bad and caspase 12, and by decreasing Bcl-2 levels expression levels. Moreover, HG treatment induced inflammation by upregulating tumor necrosis factor- $\alpha$, interleukin (IL)- $1 \beta$ and IL-6 expression. However, GAS5 and SERCA2b overexpression significantly decreased ER stress-related apoptosis and inflammation, whereas SERCA2b knockdown significantly reversed the inhibitory effect of GAS5 on ER stress, apoptosis and inflammation. The results of the present study indicated that GAS5 may suppress ER stress-induced apoptosis and inflammation by regulating SERCA2b in HG-treated cells.
\end{abstract}

Correspondence to: Dr Lei Jiang, Department of Ophthalmology, The Third People's Hospital of Wuxi, 585 Xingyuan North Road, Wuxi, Jiangsu 214000, P.R. China

E-mail: leijiang3a@163.com

Key words: growth arrest-specific transcript 5, sarcoplasmic/ endoplasmic reticulum calcium ATPase 2, endoplasmic reticulum stress, apoptosis, inflammation, diabetic retinopathy
These data suggested that GAS5 may serve a vital role in the pathogenesis of DR, and it may be considered a potential target for DR therapy.

\section{Introduction}

Diabetes is a serious and complex disease characterized by chronic hyperglycemia, which aggravates a series of metabolic reactions and increases the production of advanced glycosylation end products (AGEs). It has been reported that in diabetes the development of AGE-induced retinal inflammation results in blood-retinal barrier damage, which in turn leads to diabetic macular edema and retinal neovascularization $(1,2)$. Although blood glucose control has been reported to significantly reduce the incidence of microvascular complications, some patients still suffer from proliferative diabetic retinopathy (DR) for $>30$ years (3). Therefore, it is of vital importance to reveal the underlying mechanisms of retinal impairment in DR. Several studies have shown that the endoplasmic reticulum (ER) stress promotes inflammation and apoptosis in DR $(4,5)$. The pro-inflammatory cytokines, including tumor necrosis factor- $\alpha$ (TNF- $\alpha$ ), interleukin (IL) $-1 \beta$ and IL-6, contribute to endothelial cell impairment and disruption of blood-brain barrier, resulting in the development of macular edema (6). Furthermore, ER stress and inflammatory processes synergistically aggravate the progression of $\operatorname{DR}(7,8)$.

ER stress is induced by several pathological factors, and this is followed by aberrant accumulation of proteins. At the early stages of ER, the unfolded-protein response pathway upregulates the expression levels of transcriptases and chaperone proteins, thereby increasing the volume and enhancing the ability of protein folding in the ER for restoring its homeostasis. Protein kinase R-like ER kinase (PERK), a member of the eukaryotic initiation factor $2 \alpha(\mathrm{IF} 2 \alpha)$ protein kinase family, is activated in response to several stress stimuli. In addition, phosphorylated (p)-PERK induces the protective stress mechanism by phosphorylating eIF $2 \alpha$, which subsequently regulates the expression of activating transcription factor (ATF) proteins. When severe or sustaining ER stress causes irreversible cell damage, the apoptosis signaling pathway may be activated by CCAAT/enhancer-binding protein homologous protein (CHOP) upregulation (9). CHOP upregulation may result in the activation of ATF4, promoting the expression of apoptosis-related proteins, including Bax and 
caspase 12 (10-12). Therefore, these ER stress-related proteins (p-PERK, ATF4, CHOP, p-Eif $2 \alpha$ ) may reflect ER stress more accurately.

It has been demonstrated that the long non-coding RNA growth arrest-specific 5 (lncRNA GAS5) regulates gene expression and affects protein function. For example, a previous study revealed that IncRNA GAS5 knockdown induced glucose uptake in normal adipocytes and downregulated the expression of insulin receptor genes by binding to the promoter region (13). Other studies have shown that GAS5 expression is abnormally altered and triggers specific pathological processes in several diseases. For example, the expression of 1ncRNA GAS5 was decreased in endometrial carcinoma tissue specimens from patients with type 2 diabetes and in human endometrial carcinoma cells exposed to high glucose (HG); however, lncRNA GAS5 overexpression inhibited the proliferation of these cells (14). Furthermore, gene expression analysis based on the Gene Expression Omnibus database revealed that GAS5 was downregulated in diabetic lymphatic endothelial cells (15). These findings indicated that GAS5 served a key role in diabetes. In addition, lncRNA GAS5 has been reported to be involved in other biological processes, such as inflammation and apoptosis (16-18). Sarcoplasmic/endoplasmic reticulum $\mathrm{Ca}^{2+}$ ATPase (SERCA) is a type of ATPase that transports cations across the cell membrane with 10 transmembrane domains. SERCA2b is located at the sarcoplasmic reticulum or ER membrane of all types of cells and is responsible for maintaining intracellular $\mathrm{Ca}^{2+}$ homeostasis. It has been reported that SERCA2b regulates ER stress (19), which is attenuated in pancreatic $\beta$-cells (20). A previous study has indicated that SERCA2 exerts protective effects by regulating HG-treated ER stress and apoptosis in podocytes (19). Therefore, the present study aimed to investigate the role and function of GAS5 and SERCA2b in DR. The human adult retinal pigment epithelium 19 (ARPE-19) cell line was treated with a high concentration of glucose and was used to mimic DR in vitro (21).

\section{Materials and methods}

Cells. The ARPE-19 cell line was purchased from the American Type Culture Collection and cultured in DMEM (Gibco; Thermo Fisher Scientific, Inc.) supplemented with $10 \%$ fetal bovine serum (Gibco; Thermo Fisher Scientific, Inc.), $100 \mathrm{U} / \mathrm{ml}$ penicillin and $100 \mathrm{mg} / \mathrm{ml}$ streptomycin. Cells were incubated in a humidified atmosphere containing $5 \%$ $\mathrm{CO}_{2}$ at $37^{\circ} \mathrm{C}$. ARPE-19 cells were detached following digestion with trypsin (2.5 g/l; Gibco; Thermo Fisher Scientific, Inc.) until intercellular space enlarged, cytoplasm retracted and the cells exhibited a round morphology. These changes were observed by inverted microscopy (magnification, x20). Subsequently, the trypsin solution was centrifuged at $250 \mathrm{xg}$ for 5 min to collect cells and then cells were resuspended in DMEM medium for further experiments. Cells were divided into three groups as follows: i) Normal glucose (NG) group, which were treated with $5.5 \mathrm{mM}$ glucose; ii) osmotic control mannitol group (MG), which were treated with $5.5 \mathrm{mM}$ glucose plus $27.5 \mathrm{mM}$ mannitol, as previously described (21-23); and iii) high glucose (HG) group, which were treated with $33.3 \mathrm{mM}$ glucose. Cells were cultured for $48 \mathrm{~h}$ at $37^{\circ} \mathrm{C}$ before further experiments. The transfection experiments, described below, were performed in the $\mathrm{HG}$ group only. Following transfection, cells were further incubated at $37^{\circ} \mathrm{C}$ for $48 \mathrm{~h}$. The transfection efficiency was evaluated by reverse transcription-quantitative PCR (RT-qPCR). After transfection, cells were treated with $\mathrm{HG}$ for $48 \mathrm{~h}$ at $37^{\circ} \mathrm{C}$.

$R T-q P C R$. Total RNA was extracted from cells using TRIzol ${ }^{\circledR}$ (Beyotime Institute of Biotechnology) and reversely transcribed into cDNA using the High Capacity cDNA Reverse Transcription kit (Thermo Fisher Scientific, Inc.). Subsequently, qPCR was performed using the Maxima SYBR Green qPCR Master Mix (Thermo Fisher Scientific, Inc.) and the Real-Time PCR System (Applied Biosystems, Thermo Fisher Scientific, Inc.). The following thermocycling conditions were used for qPCR: Initial denaturation at $94^{\circ} \mathrm{C}$ for $5 \mathrm{~min}$; and 40 cycles of $95^{\circ} \mathrm{C}$ for $5 \mathrm{sec}, 65^{\circ} \mathrm{C}$ for $34 \mathrm{sec}$ and $72^{\circ} \mathrm{C}$ for $30 \mathrm{sec}$. The following primers were used for qPCR: SERCA2b, forward 5'-CGAACCCTTGCCACTCATCTTC3', reverse 5'-TGCCGA GAACGAGCAGGATTTG-3'; GAS5, forward 5'-CGACTC CTGTGAGGTATGGTG-3', reverse 5'-ATCCTTCCTTGG GGACACAAC-3'; for GADPH control, forward 5'-GGAGCG AGATCCCTCCAAAAT-3', reverse 5'-GGCTGTTGTCAT ACTTCTCATGG-3'. The relative expression of GAS5 and SERCA2b mRNA was quantified using $2^{-\Delta \Delta C q}$ method (22) and normalized to the internal reference gene GAPDH.

Western blot. Following treatment, ARPE-19 cells were lysed with RIPA buffer (Beyotime Institute of Biotechnology) supplemented with PMSF and total protein extracts were isolated. Protein concentration was quantified using a bicinchoninic acid assay. Subsequently, proteins were separated by SDS-PAGE (stacking gel 5\%; separation gel 12\%) and transferred to PVDF membranes. The membranes were blocked with $5 \%$ skim milk for $2 \mathrm{~h}$ at room temperature and incubated with primary antibodies against SERCA2B (1:1,000; cat. no. ab2861; Abcam), ATF4 (1:1,000; cat. no. ab184909; Abcam), Bcl-2 (1:1,000; cat. no. ab32124; Abcam), Bax (1:1,000; cat. no. ab32503; Abcam), total PERK [1:1,000; cat. no. 3192; Cell Signaling Technology (CST)], phosphorylated (p)-PERK (1:1,000; cat. no. 3179; CST), eIF2 $\alpha$ (1:1,000; cat. no. 2103; CST), p-eIF2 $\alpha$ (1:1,000; cat. no. 3398; CST), Bad (1:2,000; cat. no. ab32445; Abcam), caspase 12 (1:1,000; cat. no. 55238-1-AP; Proteintech), CHOP (1:1,000; cat. no. BS1136; Bioworld Technology), GADPH (1:10,000; cat. no. ab181603; Abcam) at $4^{\circ} \mathrm{C}$ overnight. On the following day, membranes were washed with tris-buffered saline + Tween-20 (1X TBST) and incubated with horseradish peroxidase (HRP)-conjugated goat anti-rabbit (1:1,000; cat. no. 31460; Thermo Fisher Scientific, Inc.) or HRP-conjugated goat anti-mouse (1:2,000; cat. no. ab47827; Abcam) secondary antibodies at room temperature for $1 \mathrm{~h}$, followed by three washes with TBST. The immunoreactive proteins were visualized using the enhanced chemiluminescence method (Advansta, America), and images captured by a gel imaging system (Gene, America). ImageJ software (version 1.46r; National Intitutes of Health) was used to determine the mean gray value, and calculate the gray value ratio between the target protein and the internal reference protein (GADPH), to indicate the relative protein expression levels. 
Plasmid transfection. GAS5 recombinant overexpression plasmid pcDNA3.1 (OE-GAS5), SERCA2b recombination plasmid (OE-SERCA2b) and the empty control plasmid (OE-NC) were purchased from Genomeditech. Short hairpin RNA (shRNA) SERCA2b plasmids (pGPU6/GFP/Neo, shRNA-SERCA2B-1 and shRNA-SERCA2B-2) and control plasmids with scrambled sequences (pGPU6/GFP/Neo; shRNA-NC) were obtained from GenePharma. Cells were seeded into the 6 -well plate $\left(1 \times 10^{5} /\right.$ well). For transfection, $200 \mu \mathrm{l}$ transfection buffer, $4 \mu \mathrm{l}$ Lipofectamine (Thermo Fisher Scientific, Inc) and $2 \mu \mathrm{g}$ plasmid were added into an eppendorf tube and mixed. Subsequently, cells were transfected with the transfection mixture for $48 \mathrm{~h}$ at $37^{\circ} \mathrm{C}$. Following the incubation, subsequent experiments were performed.

ELISA. ARPE-19 cells were homogenized at $12,000 \mathrm{x}$ g at $4^{\circ} \mathrm{C}$ for $15 \mathrm{~min}$ and the supernatants were collected using a pipette. The supernatant was analyzed using TNF- $\alpha$ (cat. no. SBJ-H0038), IL-1 $\beta$ (cat. no. SBJ-H0417) or IL-6 (cat. no. SBJ-H0456) ELISA kits (Nanjing SenBeiJia Biological Technology Co., Ltd.) according to the manufacturer's instructions.

Flow cytometry. Cells were seeded into 6-well plates $\left(1 \times 10^{5} /\right.$ well) and treated with $\mathrm{MG}, \mathrm{HG}, \mathrm{OE}-\mathrm{GAS} 5$ or shRNA-SERCA2B-1. The Annexin V-FITC/PI Cell Apoptosis kit (Invitrogen; Thermo Fisher Scientific, Inc.) was used to detect apoptotic rates. Briefly, cells of each group were digested with trypsin and washed with PBS, followed by centrifugation at $1,000 \mathrm{x} \mathrm{g}$ to discard the supernatant. Then, cells were resuspended in binding buffer (500 $\mu \mathrm{l})$ and subsequently, Annexin V-FITC was added to the cells for $15 \mathrm{~min}$ at $37^{\circ} \mathrm{C}$. Next, propidium iodide staining solution was added to the cells at room temperature for $15 \mathrm{~min}$ in the dark. Apoptotic rates were measured by flow cytometry. The scatter plot quadrants were divided as: Upper left $\left(\mathrm{FITC}^{-} / \mathrm{PI}^{+}\right)$, cell fragments that have lost their cell membranes, or dead cells from other causes; lower left (FITC'/ $/ \mathrm{PI}^{-}$), normal (living) cells; upper right $\left(\mathrm{FITC}^{+} / \mathrm{PI}^{+}\right)$, non-viable apoptotic cells; lower right $\left(\mathrm{FITC}^{+} / \mathrm{PI}^{-}\right)$, viable apoptotic cells. The cell apoptotic rate was calculated by the formula: Cell apoptotic rate = non-viable apoptotic cells + viable apoptotic cells.

Statistical analysis. The experimental data were analyzed using GraphPad Prism (version 6.0; GraphPad Software, Inc.) and presented as the mean \pm SEM. The difference among groups was analyzed with one-way ANOVA. The comparison of two groups was analyzed with post-hoc Tukey's test. $\mathrm{P}<0.05$ was considered as statistically significant. All experiments were repeated three times.

\section{Results}

GAS5 and SERCA2b expression levels decrease in HG-treated ARPE-19 cells. RT-qPCR and western blotting were used to detect lncRNA GAS5 and SERCA2b protein expression levels, respectively, after varying glucose treatments in ARPE-19 cells. There was no significant difference in GAS5 (Fig. 1A), SERCA2b protein and ER stress levels (Fig. 1B and C) between NG group and MG group, suggesting that the influence of $\mathrm{HG}$ on ARPE-19 was not due to osmotic pressure. By contrast, HG treatment reduced the levels of GAS5 and SERCA2b compared with NG and MG, indicating that GAS5 and SERCA2b may serve vital roles in cells under HG exposure. In addition, HG-induced ER stress markers were assessed by western blotting in ARPE-19 cells. The results indicated that the ratios of p-PERK/PERK and p-Eif $2 \alpha /$ Eif $2 \alpha$ were significantly increased in HG-induced cells compared with NG and MG cells. Furthermore, ATF4 and CHOP levels were increased by HG compared with NG and MG cells (Fig. 3C). The results suggested that HG significantly increased ER stress compared with NG or MG treatments (Fig. 1B and C).

Overexpression of GAS5 and SERCA2b inhibits $H G$-induced ER stress. Next, the role of GAS5 overexpression in ARPE-19 cells treated with HG was investigated. The results presented in Fig. 2A show the increased expression levels of GAS5 in OE-GAS5-transfected cells compared with the OE-NC-transfected control group, which indicated successful transfection of the overexpression vector (Fig. 2A). In addition, overexpressed GAS5 significantly increased SERCA2b protein expression levels, suggesting that GAS5 affected the expression of SERCA2b (Fig. 2B). Furthermore, compared with the control groups, upregulation of GAS5 significantly reduced the total protein expression levels of ATF4 and CHOP, as well as the relative protein phosphorylation ratio of $\mathrm{p}$-PERK/PERK and p-eIF2 $\alpha /$ IF $2 \alpha$ in ARPE-19 cells exposed to HG (Fig. 2B).

To study the role of SERCA2b in HG-treated ARPE-19 cells, a SERCA2b overexpression plasmid was used to transfect cells (Fig. 2C). SERCA2b overexpression also significantly decreased the total protein expression levels of ATF4 and CHOP, as well as the ratios of p-PERK/PERK and p-eIF2 $\alpha / \mathrm{eIF} 2 \alpha$. Thus, these data suggested that GAS5 and SERCA2b could inhibit HG-induced ER stress in vitro (Fig. 2C).

Overexpression of GAS5 reduces $H G$-induced inflammatory factors through SERCA2b. Considering that GAS5 was found to significantly alter SERCA2b expression, two SERCA2b knockdown plasmids (shRNA-SERCA2B-1 and shRNA-SERCA2B-2) were used to decrease expression of endogenous SERCA2b in ARPE-19 cells (Fig. 3A and B). The results showed that the effects of shRNA-SERCA2B-1 plasmid in reducing SERCA2b expression was more efficient than shRNA-SERCA2B-2; thus, shRNA-SERCA2B-1 plasmid was used to perform subsequent experiments. To evaluate the effects of shRNA-SERCA2B-1 plasmid on SERCA2b expression, ARPE-19 cells were co-transfected with OE-GAS5 and shRNA-SERCA2b. Western blotting and RT-qPCR results indicated that SERCA2b protein and mRNA expression levels, respectively, were significantly reduced by shRNA-SERCA2b in the presence of GAS5 overexpression plasmid, compared with the control groups (Fig. 3C and D). TNF- $\alpha$, IL-1 $\beta$ and IL- 6 concentrations were detected by ELISA and used to assess inflammation indexes in vitro (Fig. 3E). Overexpression of GAS5 significantly reduced proinflammation-related factor levels compared with the OE-NC group. However, SERCA2b gene knockdown significantly reversed the inhibitory effects of GAS5 upregulation on inflammation. Thus, GAS5 may affect inflammation by regulating SERCA2b.

Overexpression of GAS5 reduces $H G$-induced apoptosis through SERCA2b. The apoptosis levels were assessed by the 
A

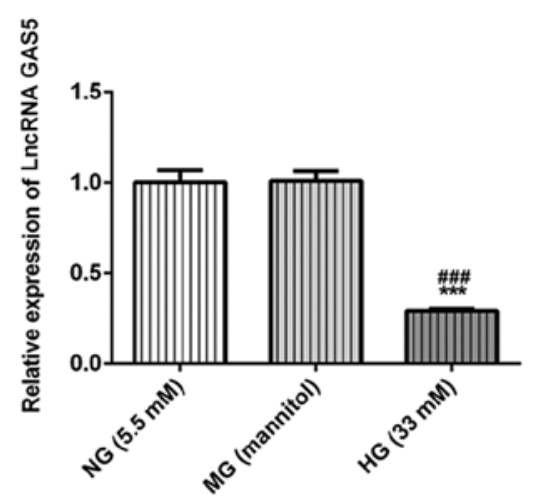

B

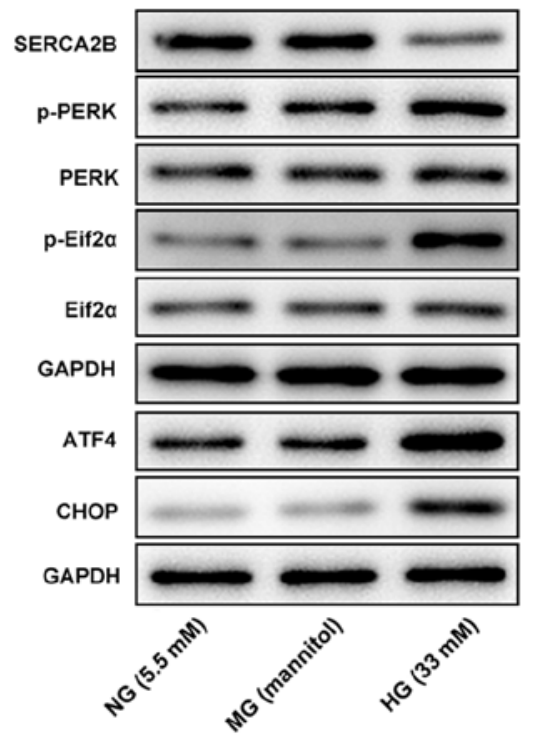

C
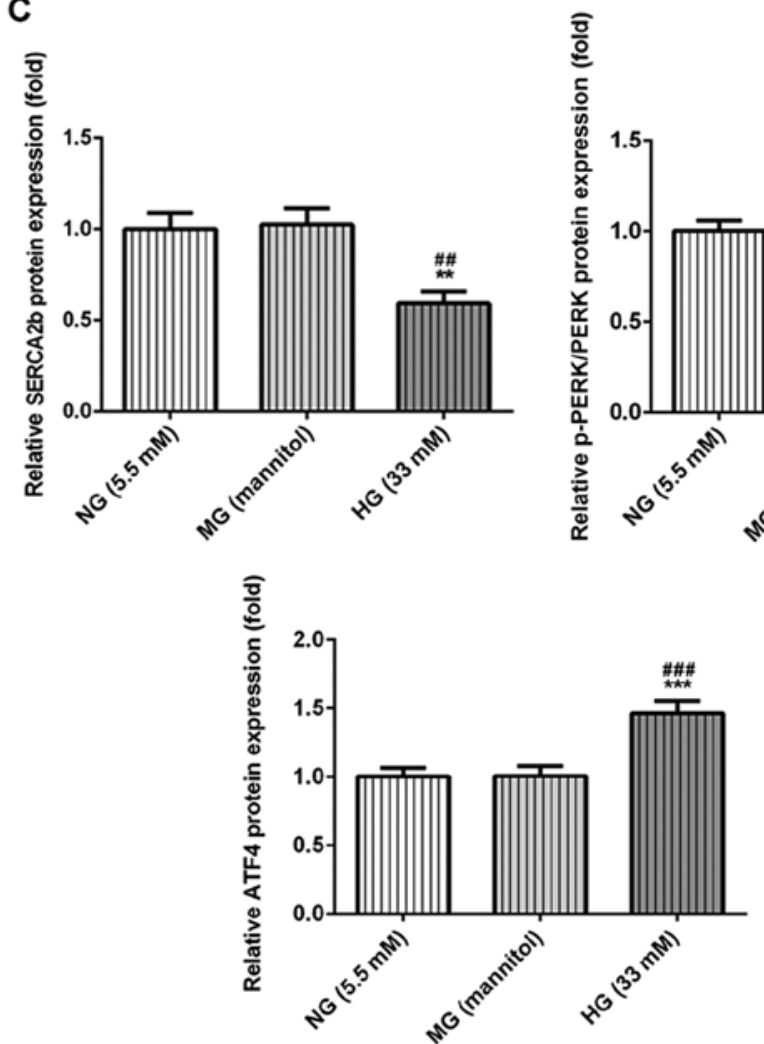
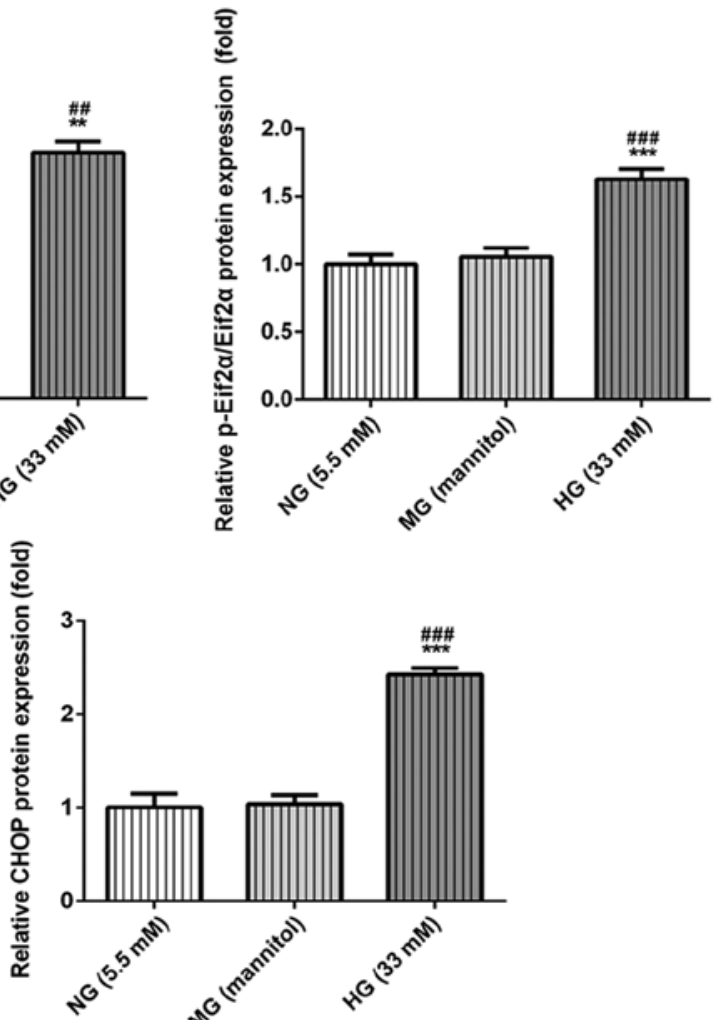

Figure 1. HG treatment decreases lncRNA GAS5 levels and increases ER stress in ARPE-19 cells. (A) Reduced lncRNA GAS5 levels in HG-treated ARPE-19 cells were determined by reverse transcription-quantitative PCR. (B) ER stress-related protein expression in HG-treated ARPE-19 cells was (B) determined by western blotting and (C) semi-quantified. ${ }^{* *} \mathrm{P}<0.01$ and ${ }^{* * * *} \mathrm{P}<0.001$ vs. NG; ${ }^{\# \#} \mathrm{P}<0.01$ and ${ }^{\# \# \#} \mathrm{P}<0.001$ vs. MG. ATF4, activating transcription factor 4 ; CHOP, CCAAT/enhancer-binding protein homologous protein; ER, endoplasmic reticulum; HG, high glucose; lncRNA GAS5, long non-coding RNA growth arrest-specific transcript 5; MG, mannitol control group; NG, normal glucose; PERK, protein kinase R-like ER kinase; SERCA2b, sarcoplasmic/endoplasmic reticulum $\mathrm{Ca}^{2+}$ ATPase 2; eIF2 $\alpha$, eukaryotic initiation factor $2 \alpha$; p phosphorylated.

measurement of apoptosis-related proteins by western blotting and flow cytometry (Fig. 4). Bcl-2, Bad, Bax and caspase 12 were used as apoptosis markers. Apoptosis levels were partially reduced by GAS5 overexpression compared with the OE-NC group, as indicated by the decreased ratio of $\mathrm{Bax} / \mathrm{Bcl} 2$ and cleaved caspase-12/pro-caspase 12, as well as the increased levels of Bad expression (Fig. 4A). Moreover, flow cytometry results indicated that GAS5 overexpression reduced the percentage of cell apoptosis in HG conditions (Fig. 4B and C). However,
SERCA2b gene knockdown was able to significantly reverse the inhibitory effects of GAS5 overexpression on cell apoptosis (Fig. 4A-C).

\section{Discussion}

In the present study lncRNA GAS5 expression levels were decreased in ARPE-19 cells treated with HG, which is consistent with previous studies that have demonstrated that HG treatment 
B

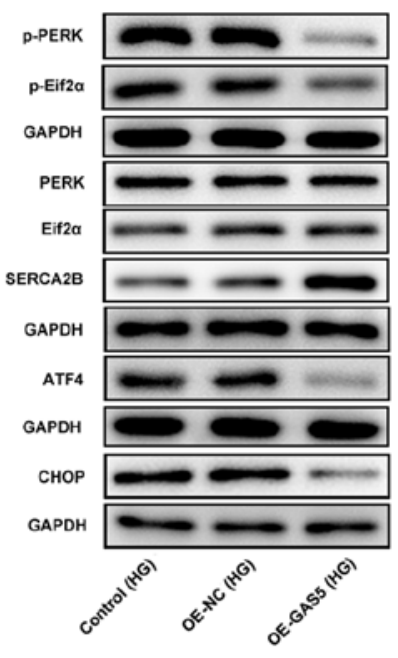

C

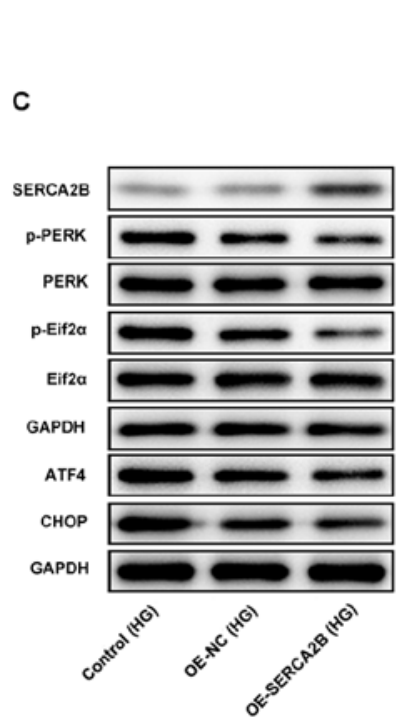

A

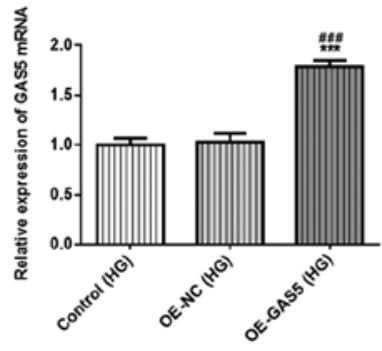

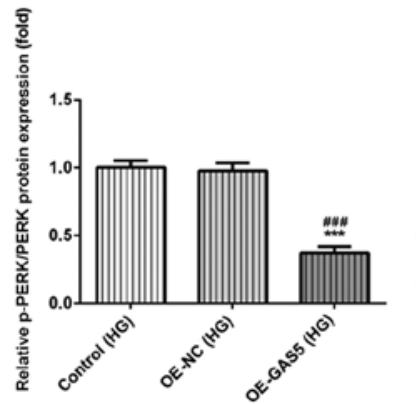
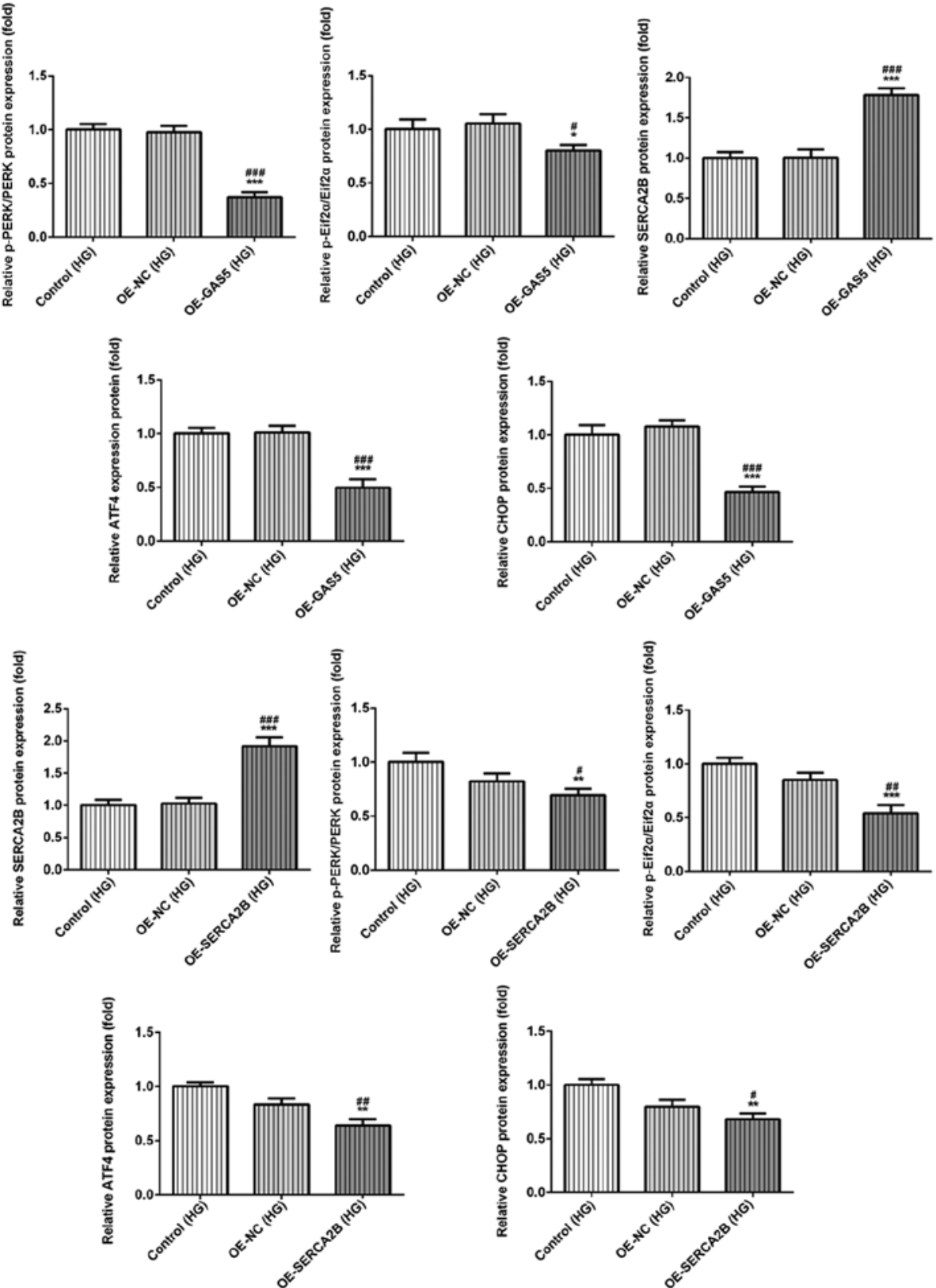

Figure 2. Overexpression of GAS5 or SERCA2b affects ER stress in HG-treated ARPE-19 cells. (A) lncRNA GAS5 expression levels were increased in cells transfected with pcDNA-GAS5, as determined by reverse transcription-quantitative PCR. (B) GAS5 overexpression upregulated SERCA2b protein expression levels and reduced ER stress. (C) SERCA2b expression and ER stress were assessed in cells transfected with pcDNA-SERCA2b. ${ }^{*} \mathrm{P}<0.05$, ${ }^{* * *} \mathrm{P}<0.01$ and ${ }^{* * * *} \mathrm{P}<0.001$ vs. OE-NC; ${ }^{\#} \mathrm{P}<0.05,{ }^{\# \#} \mathrm{P}<0.01$ and ${ }^{\# \# \#} \mathrm{P}<0.001$ vs. control. ATF4, activating transcription factor 4; CHOP, CCAAT/enhancer-binding protein homologous protein; eIF2 $\alpha$, eukaryotic initiation factor $2 \alpha$; ER, endoplasmic reticulum; GAS5, growth arrest-specific transcript 5; HG, high glucose (33 mM); NC, negative control; OE, overexpression vector; p-, phosphorylated; PERK, protein kinase R-like ER kinase; SERCA2b, sarcoplasmic/endoplasmic reticulum Ca ${ }^{2+}$ ATPase 2.

induces decreased GAS5 expression in several tissues or cells, such as endometrial carcinoma and adipocytes $(13,14)$. GAS5 overexpression significantly increased SERCA2b levels and reduced ER stress in ARPE-19 cells treated with HG, indicating a possible association between them. Several studies have provided insights on GAS5 mechanism of action. For example, GAS5 can bind to the promoter region of the insulin receptor gene to modulate its expression in nondiabetic adipocytes (13), acting as a 
A

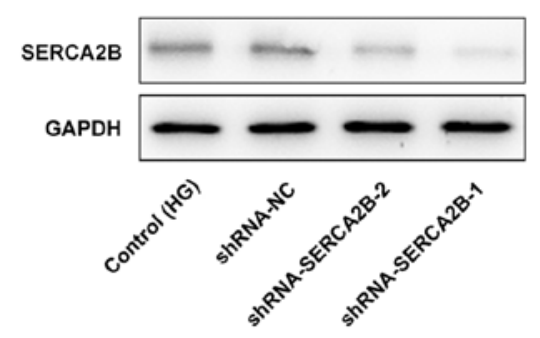

C

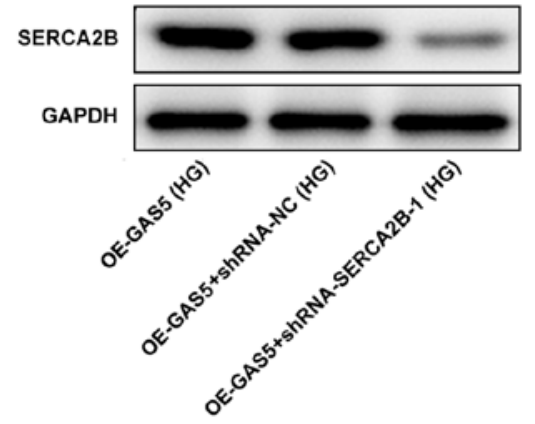

$\mathbf{E}$

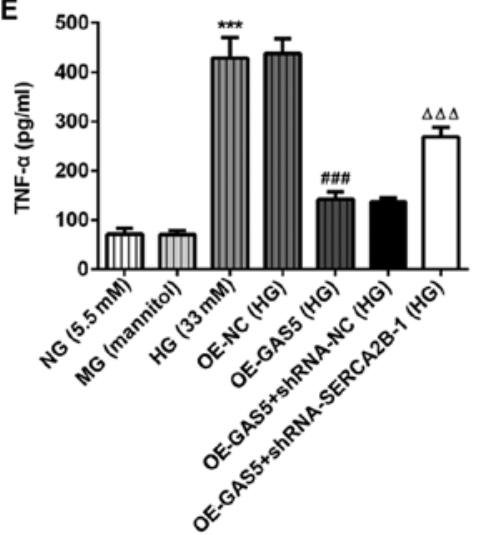

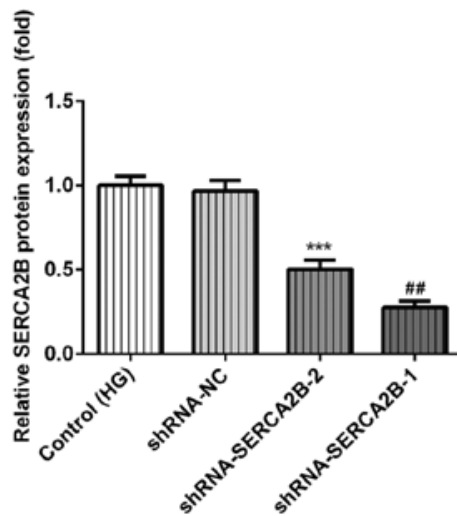

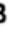
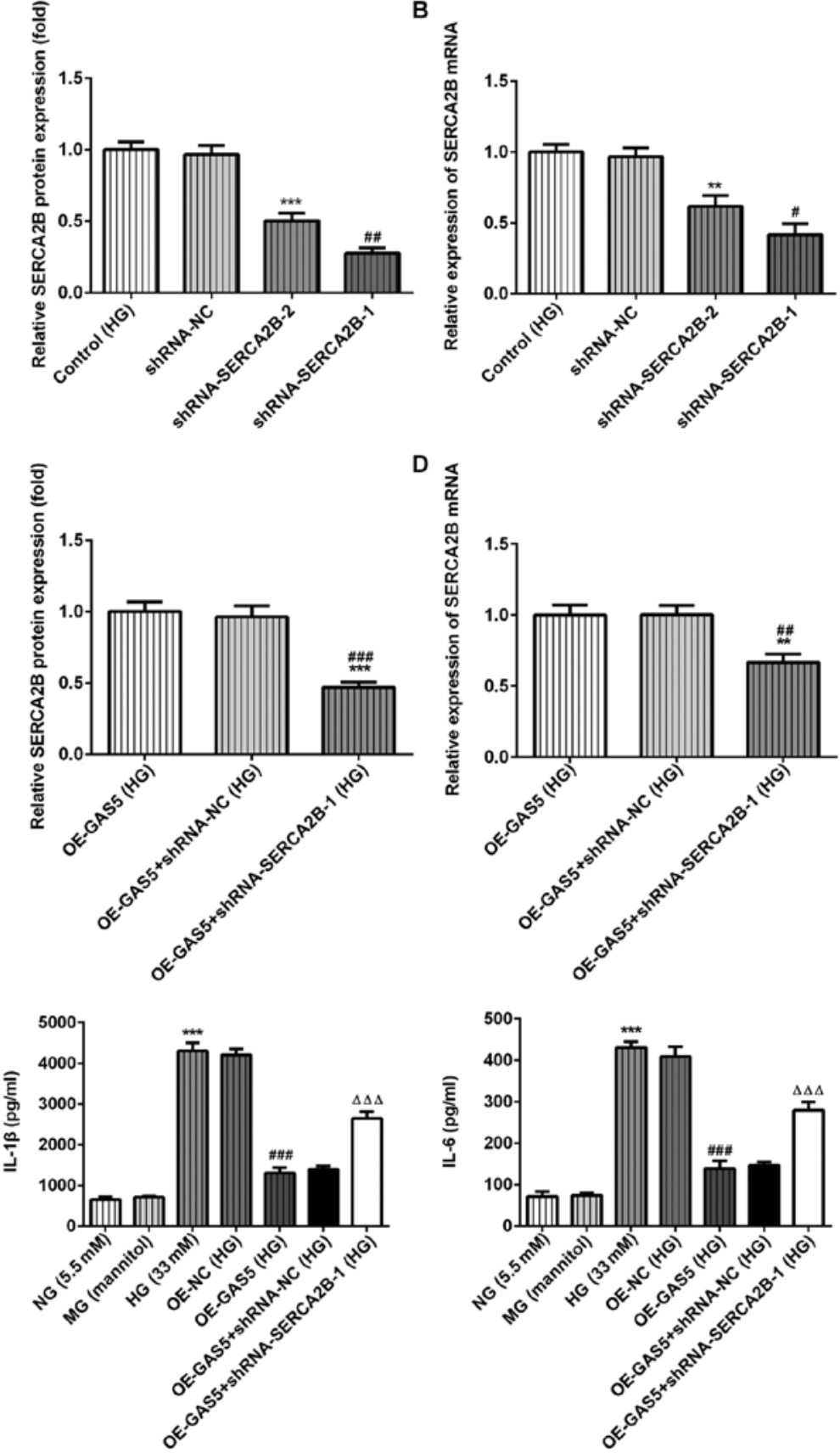

Figure 3. SERCA2b knockdown reverses the effects of GAS5 on inflammation in HG-treated ARPE-19 cells. (A and B) Evaluation of the transfection efficacy of the SERCA2b knockdown plasmids shRNA-SERCA2B-1 and shRNA-SERCA2B-2 by (A) western blotting and (B) RT-qPCR. ${ }^{* *} \mathrm{P}<0.01$ and ${ }^{* * * *} \mathrm{P}<0.001$ vs. shRNA-NC. ${ }^{*} \mathrm{P}<0.05$ and ${ }^{\# \#} \mathrm{P}<0.01$ vs. shRNA-SERCA2B-2 (C and D) SERCA2b was detected by (C) western blotting and (D) RT-qPCR after the plasmids overexpressing GAS5 and SERCA2b were co-transfected into HG-treated ARPE-19 cells. ${ }^{\# \#} \mathrm{P}<0.01$ and ${ }^{\# \# \#} \mathrm{P}<0.001$ vs. OE-GAS5; ${ }^{* *} \mathrm{P}<0.01$ and ${ }^{* * *} \mathrm{P}<0.001$ vs. OE-GAS5 + shRNA-NC. (E) The effects of GAS5 overexpression and SERCA2b knockdown on inflammatory markers. ${ }^{\Delta \Delta \Delta} \mathrm{P}<0.001$ vs. OE-GAS5 + shRNA-NC; ${ }^{\# \# \# ~} \mathrm{P}<0.001$ vs. OE-NC; ${ }^{* * * *} \mathrm{P}<0.001$ vs MG. GAS5, growth arrest-specific transcript 5; HG, high glucose; IL, interleukin; MG, mannitol control group; NC, negative control; NG, normal glucose; OE, overexpression vector; RT-qPCR, reverse transcription-quantitative PCR; SERCA2b, sarcoplasmic/endoplasmic reticulum $\mathrm{Ca}^{2+}$ ATPase 2; shRNA, short hairpin RNA; TNF- $\alpha$, tumor necrosis factor- $\alpha$.

competing endogenous RNA for microRNA (miRNA) involved in the regulation of the expression of numerous proteins involved in cancer $(23,24)$. The findings of the present study suggest that GAS5 may serve a role as a regulatory RNA or a miRNA sponge to regulate miRNA levels and affect SERCA2b expressions in DR. It has been reported that SERCA is involved in maintaining $\mathrm{Ca}^{2+}$ homeostasis in the ER, by transferring $\mathrm{Ca}^{2+}$ from the cytosol into the ER (25). Therefore, changes in SERCA2b expression levels may affect the $\mathrm{Ca}^{2+}$ concentration, which in turn affects
$\mathrm{Ca}^{2+}$-mediated signaling pathways, resulting in abnormal metabolic processes, such as lipid metabolism (26). The eleventh transmembrane helix (TM11) of SERCA2b fine-tunes the intramolecular interactions with other transmembrane regions to modulate SERCA2b activity (27). The modulation of TM11 plays a key role in maintaining $\mathrm{Ca}^{2+}$ homeostasis in the ER. Recent studies have suggested that ER-resident proteins can regulate SERCA2b activity (28-30). The exact mechanism by which these proteins affect the location of TM11 or its interaction with other 
A

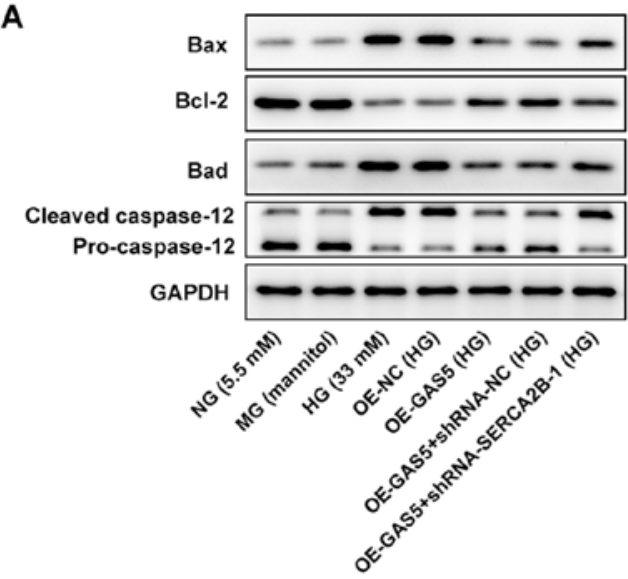

в
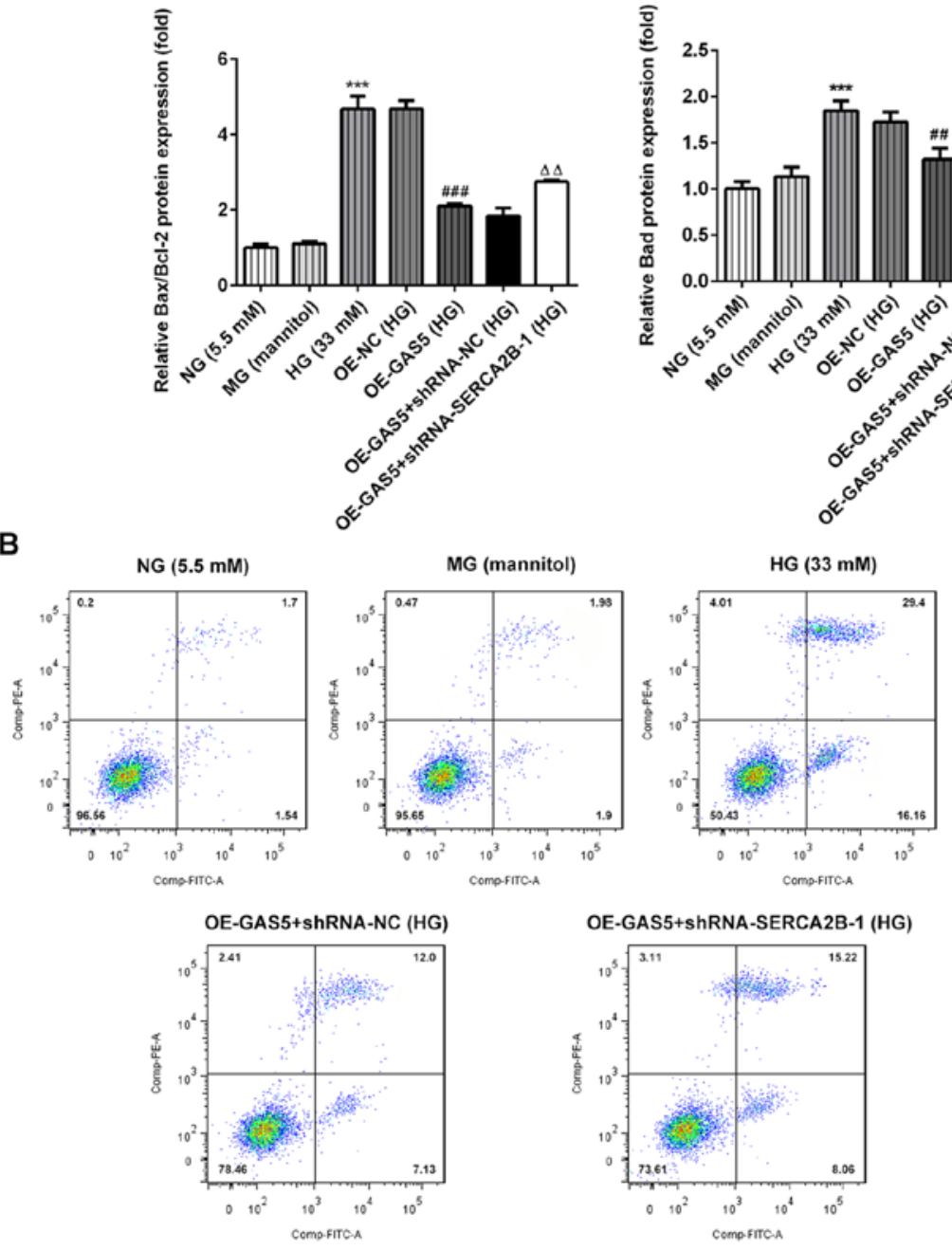
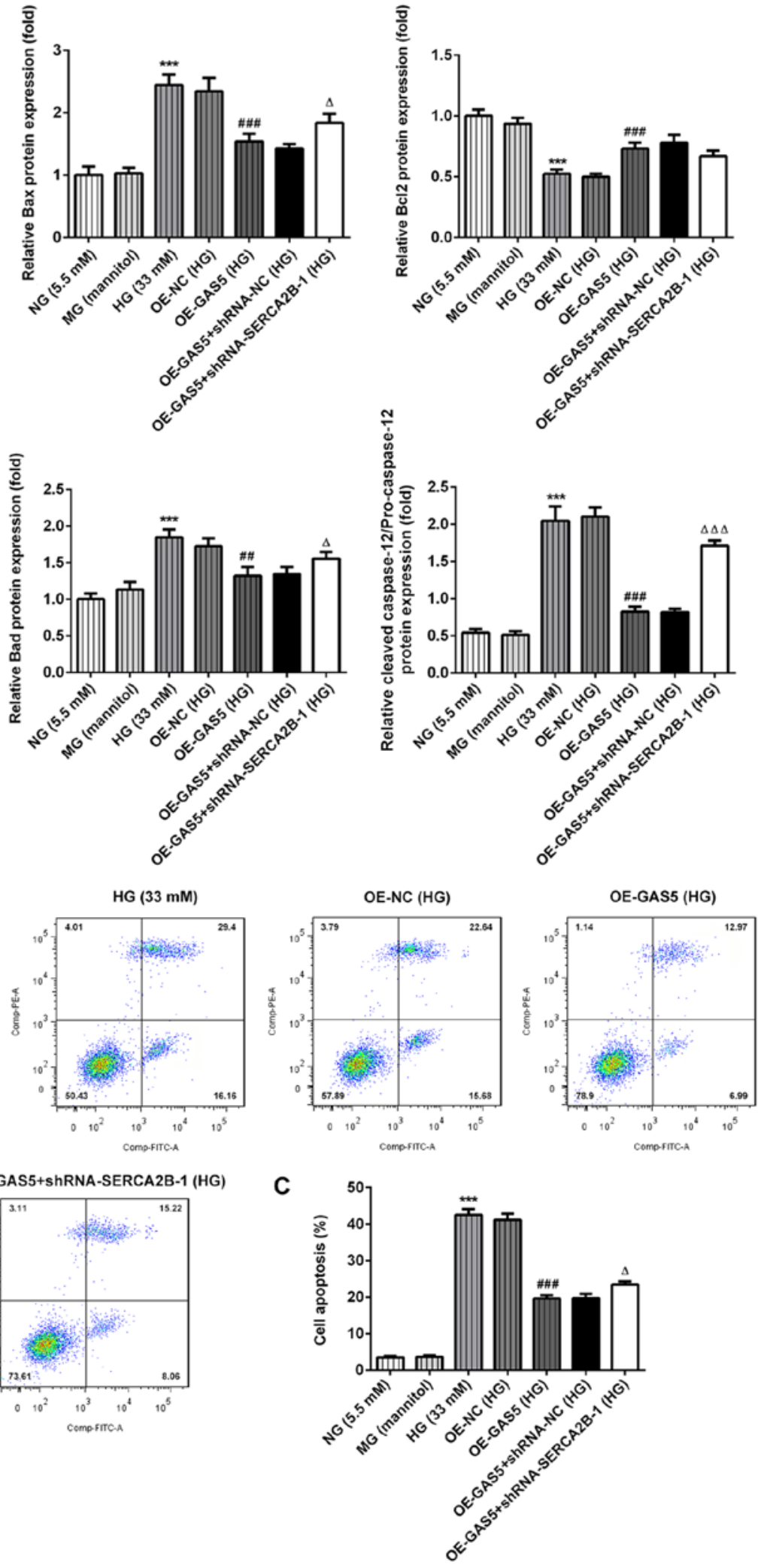

Figure 4. SERCA2b reverses the effects of GAS5 on apoptosis in HG-treated ARPE-19 cells. (A) apoptosis-related protein expression levels were detected by western blotting. (B and C) Flow cytometry was used to determine apoptotic rates. ${ }^{\# \#} \mathrm{P}<0.01$ and ${ }^{\# \# \#} \mathrm{P}<0.001$ vs. OE-NC; ${ }^{* * *} \mathrm{P}<0.001$ vs. MG; ${ }^{\Delta} \mathrm{P}<0.05{ }^{\Delta \Delta} \mathrm{P}<0.01$ and ${ }^{\Delta \Delta \Delta} \mathrm{P}<0.001$ vs. OE-GAS5 + shRNA-NC. GAS5, growth arrest-specific transcript 5; HG, high glucose; MG, mannitol control group; NG, normal glucose; OE, overexpression vector; SERCA2b, sarcoplasmic/endoplasmic reticulum $\mathrm{Ca}^{2+}$ ATPase 2; shRNA-NC, negative control; shRNA, short hairpin RNA.

regions of SERCA2b have not yet been fully addressed (28-30). These results indicate a possible mutual influence between ER stress and SERCA2b activity; however, additional studies are required to further confirm these findings. Calpain is activated in response to abnormal $\mathrm{Ca}^{2+}$ levels in the cytosol and subsequently promotes caspase 12 activation, inducing apoptosis (31). 
Therefore, it is possible that SERCA2b overexpression inhibited ER stress-induced apoptosis by altering $\mathrm{Ca}^{2+}$ levels in $\mathrm{HG}$-treated ARPE-19 cells in the present study. However, this mechanism should be further investigated.

lncRNA GAS5 overexpression increased anti-apoptotic Bcl-2 and decreased pro-apoptotic Bad and Bax protein expression levels. In addition, SERCA2b knockdown significantly reversed the inhibitory effects of GAS5 during inflammation and apoptosis, indicating that GAS5 affected the induction of these two processes by HG partially through SERCA2b. Caspase 12 is located in the ER and is a major protein involved in ER stress-induced apoptosis $(32,33)$. Caspase 12 is caused by specific stimuli, such as higher calcium, hypoxia and lower glucose levels $(32,33)$. Moreover, downregulation of caspase 12 expression attenuated methylglyoxal-modified collagen-induced apoptosis (34). The results of the present study indicated that SERCA2b and GAS5 mediated a decrease in CHOP protein expression. Previous studies have indicated that the CHOP-mediated apoptotic rate was reduced partially by increased Bcl-2 and decreased Bad, Bax and caspase 12 levels $(11,35)$. These findings suggested that GAS5 attenuated ER stress-induced apoptosis through SERCA2b. The imbalance of the expression of pro-apoptotic and anti-apoptotic proteins of the Bcl-2 family may contribute to mitochondrial damage and subsequently activate caspases, inducing cell apoptosis via the intrinsic apoptosis pathway (36). Among the Bcl-2 family members, Bcl-2 is involved in preserving mitochondrial integrity, which may be disrupted by Bax (37). Furthermore, flow cytometry results from the present study showed that apoptosis was significantly reduced by GAS5 overexpression, suggesting that changes on these apoptosis-related proteins might lead to reduced apoptosis levels. There are certain mechanisms involved in cell apoptosis, one of which was ER stress-mediated apoptosis. On the one hand, phosphorylation of eIF2 $\alpha$ induced by PERK could decrease protein synthesis to reduce ER stress; on the other hand, it also could mediate CHOP expression to induce apoptosis (38). The current study indicated that GAS5 affected inflammatory markers partly through SERCA2b.

Previous studies showed that ER stress induced cell apoptosis and inflammation $(39,40)$. Inflammation in DR is facilitated through increasing ER stress (41), suggesting that HG-induced ER stress may promote inflammatory response. Results from the present study may aid our further understanding of the mechanism of retinal pigment epithelium cell impairment induced by HG. Moreover, shows obvious activation in retinal of mice with DR or in retinal cell lines exposed to HG. Retinal cell damage can be alleviated through inhibiting ER stress (42-44). Thus, GAS5/SERCA2b could be potential targets for treating DR.

In conclusion, HG downregulated GAS5 expression, followed by a decrease in SERCA2b expression levels., Furthermore, HG enhanced ER stress-related apoptosis and pro-inflammatory factors, and induced cell apoptosis in ARPE-19 cells. Therefore, the results indicated that GAS5 regulated ER stress-related apoptosis and inflammation in retinal epithelial cells via SERCA2b. LncGAS5 and SERCA2b may serve as potential therapeutic targets for DR.

\section{Acknowledgements}

Not applicable.

\section{Funding}

No funding was received.

\section{Availability of data and materials}

The datasets used and/or analyzed during the current study are available from the corresponding author on reasonable request.

\section{Authors' contributions}

LJ, CW and XS conceived and designed the study, collected, analysed and interpreted the data, and revised the manuscript. LJ wrote the manuscript. All authors read and approved the final manuscript.

\section{Ethics approval and consent to participate}

Not applicable.

\section{Patient consent for publication}

Not applicable.

\section{Competing interests}

The authors declare that they have no competing interests.

\section{References}

1. Kumari N, Karmakar A and Ganesan SK: Targeting epigenetic modifications as a potential therapeutic option for diabetic retinopathy. J Cell Physiol 235: 1933-1947, 2020.

2. Leal EC, Manivannan A, Hosoya K, Terasaki T, Cunha-Vaz J, Ambrosio AF and Forrester JV: Inducible nitric oxide synthase isoform is a key mediator of leukostasis and blood-retinal barrier breakdown in diabetic retinopathy. Invest Ophthalmol Vis Sci 48: 5257-5265, 2007.

3. Diabetes Control and Complications Trial/Epidemiology of Diabetes Interventions and Complications (DCCT/EDIC) Research Group; Nathan DM, Zinman B, Cleary PA, Backlund JY, Genuth S, Miller R and Orchard TJ: Modern-day clinical course of type 1 diabetes mellitus after 30 years' duration: The diabetes control and complications trial/epidemiology of diabetes interventions and complications and pittsburgh epidemiology of diabetes complications experience (1983-2005). Arch Intern Med 169: 1307-1316, 2009.

4. Elmasry K, Ibrahim AS, Saleh H, Elsherbiny N, Elshafey S, Hussein KA and A1-Shabrawey M: Role of endoplasmic reticulum stress in 12/15-lipoxygenase-induced retinal microvascular dysfunction in a mouse model of diabetic retinopathy. Diabetologia 61: 1220-1232, 2018.

5. Wang S, Liu Y, Tan JW, Hu T, Zhang HF, Sorenson CM, Smith JA and Sheibani N: Tunicamycin-induced photoreceptor atrophy precedes degeneration of retinal capillaries with minimal effects on retinal ganglion and pigment epithelium cells. Exp Eye Res 187: 107756, 2019.

6. Rubsam A, Parikh S and Fort PE: Role of inflammation in diabetic retinopathy. Int J Mol Sci 19: E942, 2018.

7. Hotamisligil GS: Endoplasmic reticulum stress and the inflammatory basis of metabolic disease. Cell 140: 900-917, 2010.

8. Zhang K and Kaufman RJ: From endoplasmic-reticulum stress to the inflammatory response. Nature 454: 455-462, 2008.

9. Yang C, Diiorio P, Jurczyk A, O'Sullivan-Murphy B, Urano F and Bortell R: Pathological endoplasmic reticulum stress mediated by the IRE1 pathway contributes to pre-insulitic beta cell apoptosis in a virus-induced rat model of type 1 diabetes. Diabetologia 56: 2638-2646, 2013 
10. Guo HL, Hassan HM, Ding PP, Wang SJ, Chen X, Wang T, Sun LX, Zhang LY and Jiang ZZ: Pyrazinamide-induced hepatotoxicity is alleviated by 4-PBA via inhibition of the PERK-eIF2 $\alpha$-ATF4-CHOP pathway. Toxicology 378: 65-75, 2017.

11. Wang Q, Yuan X, Chen Y,Zheng Q, Xu L and Wu Y: Endoplasmic reticulum stress mediated MDRV p10.8 protein-induced cell cycle arrest and apoptosis through the PERK/eIF2 $\alpha$ pathway. Front Microbiol 9: 1327, 2018

12. Shi Y, Parag S, Patel R, Lui A, Murr M, Cai J and Patel NA Stabilization of lncRNA GAS5 by a small molecule and its implications in diabetic adipocytes. Cell Chem Biol 26: 319-330. e316, 2019.

13. Li Z, Yu Z, Meng X, Zhou S, Xiao S, Li X, Liu S and Yu P: Long noncoding RNA GAS5 impairs the proliferation and invasion of endometrial carcinoma induced by high glucose via targeting miR-222-3p/p27. Am J Transl Res 11: 2413-2421, 2019.

14. Qi M, Zhou Q, Zeng W, Shen M, Liu X, Luo C, Long J, Chen W, Zhang J and Yan S: Analysis of long non-coding RNA expression of lymphatic endothelial cells in response to type 2 diabetes. Cell Physiol Biochem 41: 466-474, 2017.

15. Xie X, Dai J, Huang X, Fang C and He W: MicroRNA-145 inhibits proliferation and induces apoptosis in human prostate carcinoma by upregulating long non-coding RNA GAS5. Oncol Lett 18: 1043-1048, 2019.

16. He X, Wang S, Li M, Zhong L, Zheng H, Sun Y, Lai Y, Chen X, Wei G, Si X, et al: Long noncoding RNA GAS5 induces abdominal aortic aneurysm formation by promoting smooth muscle apoptosis. Theranostics 9: 5558-5576, 2019.

17. Salemi M, Cannarella R, Condorelli RA, Cimino L, Ridolfo F, Giurato G, Romano C, La Vignera S and Calogero AE: Evidence for long noncoding RNA GAS5 up-regulationin patients with Klinefelter syndrome. BMC Med Genet 20: 4, 2019.

18. Guo H, Wang Y, Zhang X, Zang Y, Zhang Y, Wang L, Wang H, Wang Y, Cao A and Peng W: Astragaloside IV protects against podocyte injury via SERCA2-dependent ER stress reduction and AMPK $\alpha$-regulated autophagy induction in streptozotocin-induced diabetic nephropathy. Sci Rep 7: 6852, 2017.

19. Zickri MB, Aboul-Fotouh GI, Omar AI, El-Shafei AA and Reda AM: Effect of stem cells and gene transfected stem cells therapy on the pancreas of experimentally induced type 1 diabetes. Int J Stem Cells 11: 205-215, 2018.

20. Capellades J, Navarro M, Samino S, Garcia-Ramirez M, Hernandez C, Simo R, Vinaixa M and Yanes O: geoRge: A computational tool to detect the presence of stable isotope labeling in LC/MS-based untargeted metabolomics. Anal Chem 88: 621-628, 2016.

21. Civan MM, Marano CW, Matschinsky FW and Peterson-Yantorno K: Prolonged incubation with elevated glucose inhibits the regulatory response to shrinkage of cultured human retinal pigment epithelial cells. J Membr Biol 139: 1-13, 1994.

22. Lei J, Zhao L, Zhang Y, Wu Y and Liu Y: High glucose-induced podocyte injury involves activation of mammalian target of rapamycin (mTOR)-induced endoplasmic reticulum (ER) stress. Cell Physiol Biochem 45: 2431-2443, 2018.

23. Ran Z, Zhang Y, Wen X and Ma J: Curcumin inhibits high glucose-induced inflammatory injury in human retinal pigment epithelial cells through the ROS-PI3K/AKT/mTOR signaling pathway. Mol Med Rep 19: 1024-1031, 2019.

24. Liu B, Wu S, Ma J, Yan S, Xiao Z, Wan L, Zhang F, Shang M and Mao A: IncRNA GAS5 reverses EMT and tumor stem cell-mediated gemcitabine resistance and metastasis by targeting miR-221/SOCS3 in pancreatic cancer. Mol Ther Nucleic Acids 13: 472-482, 2018.

25. Chen X, Yang C, Xie S and Cheung E: Long non-coding RNA GAS5 and ZFAS1 are prognostic markers involved in translation targeted by miR-940 in prostate cancer. Oncotarget 9: 1048-1062, 2017.

26. Ali ES, Rychkov GY and Barritt GJ: Deranged hepatocyte intracellular $\mathrm{Ca}(2+)$ homeostasis and the progression of non-alcoholic fatty liver disease to hepatocellular carcinoma. Cell Calcium 82 102057,2019

27. Inoue M, Sakuta N, Watanabe S, Zhang Y, Yoshikaie K, Tanaka Y, Ushioda R, Kato Y, Takagi J, Tsukazaki T, et al: Structural basis of sarco/endoplasmic reticulum $\mathrm{Ca}(2+)$-ATPase $2 \mathrm{~b}$ regulation via transmembrane helix interplay. Cell Rep 27: 1221-1230.e1223, 2019.
28. Marino M,Stoilova T, Giorgi C,Bachi A,Cattaneo A,Auricchio A, Pinton $P$ and Zito E: SEPN1, an endoplasmic reticulum-localized selenoprotein linked to skeletal muscle pathology, counteracts hyperoxidation by means of redox-regulating SERCA2 pump activity. Hum Mol Genet 24: 1843-1855, 2015.

29. Raturi A, Gutierrez T, Ortiz-Sandoval C, Ruangkittisakul A, Herrera-Cruz MS, Rockley JP, Gesson K, Ourdev D, Lou PH, Lucchinetti E, et al: TMX1 determines cancer cell metabolism as a thiol-based modulator of ER-mitochondria $\mathrm{Ca} 2+$ flux. J Cell Biol 214: 433-444, 2016.

30. Ushioda R, Miyamoto A, Inoue M, Watanabe S, Okumura M, Maegawa KI, Uegaki K, Fujii S, Fukuda Y, Umitsu M, et al: Redox-assisted regulation of $\mathrm{Ca} 2+$ homeostasis in the endoplasmic reticulum by disulfide reductase ERdj5. Proc Natl Acad Sci USA 113: E6055-E6063, 2016.

31. Sundar Rajan S, Srinivasan V, Balasubramanyam M and Tatu U: Endoplasmic reticulum (ER) stress diabetes. Indian J Med Res 125: 411-424, 2007.

32. Nakagawa $T$ and Yuan J: Cross-talk between two cysteine protease families. Activation of caspase- 12 by calpain in apoptosis. J Cell Biol 150: 887-894, 2000.

33. Nakagawa T, Zhu H, Morishima N, Li E, Xu J, Yankner BA and Yuan J: Caspase-12 mediates endoplasmic-reticulum-specific apoptosis and cytotoxicity by amyloid-beta. Nature 403: 98-103, 2000.

34. Nowotny K, Castro JP, Hugo M, Braune S, Weber D, Pignitter M, Somoza V, Bornhorst J, Schwerdtle T and Grune T: Oxidants produced by methylglyoxal-modified collagen trigger ER stress and apoptosis in skin fibroblasts. Free Radic Biol Med 120: 102-113, 2018.

35. Du Z, Xu S, Hu S, Yang H, Zhou Z, Sidhu K, Miao Y, Liu Z, Shen W, Reiter RJ, et al: Melatonin attenuates detrimental effects of diabetes on the niche of mouse spermatogonial stem cells by maintaining Leydig cells. Cell Death Dis 9: 968, 2018.

36. Campbell GR, To RK and Spector SA: TREM-1 Protects HIV-1-infected macrophages from apoptosis through maintenance of mitochondrial function. Mbio 10: 2019.

37. Donovan $\mathrm{M}$ and Cotter TG: Control of mitochondrial integrity by $\mathrm{Bcl}-2$ family members and caspase-independent cell death. Biochim Biophys Acta 1644: 133-147, 2004

38. Li K, Zhang L, Xiang X, Gong S, Ma L, Xu L, Wang G, Liu Y, Ji X, Liu S, et al: Arsenic trioxide alleviates airway hyperresponsiveness and promotes apoptosis of CD4+ T lymphocytes: Evidence for involvement of the ER stress-CHOP pathway. Ir J Med Sci 182: 573-583, 2013.

39. Zhou Y, Dong B, Kim KH, Choi S, Sun Z, Wu N, Wu Y, Scott J and Moore DD: Vitamin D receptor activation in liver macrophages protects against hepatic endoplasmic reticulum stress in mice. Hepatology 71: 1453-1466, 2020.

40. Zhang L, Wang Y, Zhang L, Xia X, Chao Y, He R, Han C and Zhao W: ZBTB7A, a miR-663a target gene, protects osteosarcoma from endoplasmic reticulum stress-induced apoptosis by suppressing LncRNA GAS5 expression. Cancer Lett 448: 105-116, 2019.

41. Wang Y, Wang L, Guo H, Peng Y, Nie D, Mo J and Ye L: Knockdown of MALAT1 attenuates high-glucose-induced angiogenesis and inflammation via endoplasmic reticulum stress in human retinal vascular endothelial cells. Biomed Pharmacother 124: 109699, 2020.

42. Liu J, Wei L, Wang Z, Song S, Lin Z, Zhu J, Ren X and Kong L: Protective effect of Liraglutide on diabetic retinal neurodegeneration via inhibiting oxidative stress and endoplasmic reticulum stress. Neurochem Int 133: 104624, 2020.

43. Wu S, Zhu X, Guo B, Zheng T, Ren J, Zeng W, Chen X and Ke M: Unfolded protein response pathways correlatively modulate endoplasmic reticulum stress responses in rat retinal muller cells. J Ophthalmol 2019: 9028483, 2019.

44. Lenin R, Nagy PG, Alli S, Rao VR, Clauss MA, Kompella UB and Gangaraju R: Critical role of endoplasmic reticulum stress in chronic endothelial activation-induced visual deficits in tie2-tumor necrosis factor mice. J Cell Biochem 119: 8460-8471, 2018. 